\title{
WEAKLY COMPACTLY GENERATED FRECHET SPACES
}

\section{SURJIT SINGH KHURANA}

\author{
Department of Mathematics \\ The University of Iowa \\ Iowa City, Iowa 52242 \\ U.S.A.
}

(Received on March 12, 1979 and in revised form July 13, 1979)

ABSTRACT. It is proved that a weakly compact generated Frechet space is Lindelöf in the weak topology. As a corollary it is proved that for a finite measure space every weakly measurable function into a weakly compactly generated Frechet space is weakly equivalent to a strongly measurable function. 1980 MATHEMATICS SUBJECT CLASSIFICATION CODES. Primary 28A40, 60B05, Secondary 28A20, 46A99, 54E50.

KEYWORDS AND PRHASES. Lindelöf spaces, weakly measurable functions, strongly measurable functions, universally measurable functions, Frechet spaces, weakly compact sets.

1. INTRODUCTION.

If $\mathrm{E}$ is a weakly compactly generated Banach space then it is proved in [7] that E, with weak topology, is Lindelöf. (A topological space is said to be Lindelöf if its every open covering has a countable subcovering.) In this note we extend this result to the case when $E$ is a weakly compactly generated 
Frechet space. Also, some consequences are obtained. All locally convex spaces are taken over the field of real numbers. By a Frechet space we mean a Hausdorff, metrizable, complete locally convex space; we use the notations of [4] for locally convex spaces. $E^{\prime}$ will always denote the topological dual of a locally convex space E. A locally convex space is said to be weakly compactly generated if there exists an increasing sequence of $\sigma\left(E, E^{\prime}\right)$-compact subsets of $E$ whose union is dense in $E$.

THEOREM 1. Let $\mathbf{E}$ be a weakly compactly generated Frechet space. Then $\left(E, \sigma\left(E, E^{\prime}\right)\right)$ is a Lindelöf space and $E$ is a Borel subset of $\left(E^{\prime \prime}, \sigma\left(E^{\prime \prime}, E^{\prime}\right)\right)$, $E^{\prime \prime}$ being the bidual of $\mathbf{E}$.

PROOF. Let $\left\{\mathrm{v}_{\mathrm{n}}\right\}$ be a sequence of $0-n b d$. base having the properties:

(i) each $V_{n}$ is absolutely convex and closed,

(ii) $(n+1) v_{n+1} \subset v_{n}$, for every $n$.

We take $\left\{A_{n}\right\}$ for an increasing sequence of weakly compact, absolutely convex subsets of $E$ such that $\bigcup_{n=1}^{\infty} A_{n}=H$ is dense in $E$. We identify $\left(E, \sigma\left(E, E^{\prime}\right)\right)$ as a subspace of $R^{E^{\prime}}$, with product topology. $R^{E^{\prime}}$ is a subset of the compact Hausdorff space $\bar{R}^{E^{\prime}}$, where $\bar{R}=[-\infty, \infty]$. For an $x \in R^{E^{\prime}}$ and $y \in \bar{R}^{E^{\prime}}$, $x+y \in \bar{R}^{E^{\prime}}$ has the natural meaning. For a compact set $A \subset R^{E^{\prime}}$ and a compact set $B \subset \overline{R^{\prime}}, A+B$ is compact. Thus $A_{k}+\bar{V}_{n}$ is a compact subset of $\bar{R}^{\prime}$ for each $k$ and $n, \bar{V}_{n}$ being the closure of $V_{n}$ in $\bar{R}^{\prime}$. We claim that $\bigcap_{n=1}^{\infty}\left(H+\bar{V}_{n}\right)=E$. Since $H$ is dense in $E$ and $V_{n}$ is a $0-n b d ., H+V_{n} \supset E$ for every $n$ and so $\bigcap_{n=1}^{\infty}\left(H+\bar{V}_{n}\right) \supset E$. Conversely, take an $x \in \bigcap_{n=1}^{\infty}\left(H+\bar{V}_{n}\right)$. This means there exists a sequence $\left\{h_{n}\right\} \subset H$ and a sequence $\left\{z_{n}\right\}$ with $z_{n} \in \bar{v}_{n}$ for each $n$, such that $x=h_{n}+z_{n}$ for each $n$. Fix $n_{0} \in N$ and $\epsilon>0$. Choose an $n_{l}>\max \left(n_{0}, \frac{l}{\epsilon}\right)$ and take an $n>n_{1}$. Since $v_{n_{0}} \supset n v_{n},\left|f\left(z_{n}\right)\right| \leq \frac{1}{n}<\frac{l}{n_{l}}<\varepsilon$, for every $f \in{\stackrel{\circ}{n_{0}}}_{0}$ the polar of $\mathrm{V}_{\mathrm{n}_{0}}([4])$. Thus $\mathrm{f}\left(\mathrm{x}-\mathrm{h}_{\mathrm{n}}\right) \rightarrow 0$, uniformly for $\mathrm{f} \in \stackrel{\circ}{\mathrm{V}}_{\mathrm{n}_{0}}$. From this it follows that $\left\{h_{n}\right\}$ is Cauchy in $E$ which is complete. If $h_{n} \rightarrow y$ in $E$ it 
is easy to verify that, as elements of $\overline{\mathrm{R}}^{\prime}, \mathrm{x}=\mathrm{y}$. This proves the claim. Thus, in weak topology, $E=\bigcap_{n=1}^{\infty} \bigcup_{k=1}^{\infty}\left(A_{k}+\bar{V}_{n}\right)$ is analytic and so is Lindelöf ([6]). Also $\left(E^{\prime \prime}, \sigma\left(E^{\prime \prime}, E^{\prime}\right)\right)$ can be considered as a subspace of $R^{E^{\prime}}$. Since $\left(\mathrm{A}_{\mathrm{K}}+\overline{\mathrm{V}}_{\mathrm{n}}\right)$ is compact in $\overline{\mathrm{R}}^{\prime}, \quad\left(\mathrm{A}_{\mathrm{K}}+\overline{\mathrm{V}}_{\mathrm{n}}\right) \cap \mathrm{E}^{\prime \prime}$ is closed in $\left(\mathrm{E}^{\prime \prime}, \sigma\left(\mathrm{E}^{\prime \prime}, \mathrm{E}^{\prime}\right)\right)$ and so $\left(H+\bar{V}_{n}\right) \cap E^{\prime \prime}$ is Borel in $\left(E^{\prime \prime}, \sigma\left(E^{\prime \prime}, E^{\prime}\right)\right)$. Since $E=\bigcap_{n=1}^{\infty}\left(H+\bar{V}_{n}\right) \cap E^{\prime \prime}$, it follows that $E$ is Borel in $\left(E^{\prime \prime}, \sigma\left(E^{\prime \prime}, E^{\prime}\right)\right)$.

REMARK. Similar results for Banach spaces are proved in [2, Cor. 3.2]. In the following result, some results and notations of ([3]) are used. Let $(X, \mathfrak{A}, \mu)$ be a finite measure space, E a Hausdorff locally convex space. A function $f: X \rightarrow E$ is called weakly measurable if $h \circ f$ is $\mu$-measurable for every $h \in E^{\prime}$. It is proved in ([2]) that if $f: X \rightarrow E$ is weakly measurable that the image measure $\mu: B \rightarrow R, \nu(B)=\mu\left(f^{-1}(B)\right)$, is a Baire measure on $\left(E, \sigma\left(E, E^{\prime}\right)\right), \beta$ being the class of all Baire subsets of $\left(E^{\prime}, \sigma\left(E, E^{\prime}\right)\right)$ ([2], [8]). Two weakly measurable functions $f_{i}: X \rightarrow E, i=1,2$ are said to be weakly equivalent if $h \circ f_{1}=h \circ f_{2}$ a.e. [ $\left.\mu\right]$, for every $h \in E^{\prime}$. If $E$ is Frechet then $f: X \rightarrow E$ is called strongly measurable if there exists a sequence $\left\{f_{n}\right\}$ of U-simple functions, $f_{n}: X \rightarrow E$, such that $f_{n} \rightarrow f$, pointwise a.e. $[\mu]$.

COROLLARY 2. Let $(X, \mathfrak{A}, \mu)$ be a finite measure space, $E$ a weakly compactly generated Frechet space, and $\mathrm{f}: \mathrm{X} \rightarrow \mathrm{E}$ a weakly measurable function. Then $f$ is weakly equivalent to a strongly measurable function.

PROOF. By ([3], Cor. 5) it is enough to show that image Baire measure on $\left(E, \sigma\left(E, E^{\prime}\right)\right)$ is tight (cf. [2]). Since $\left(E, \sigma\left(E, E^{\prime}\right)\right)$ is Lindelöf, Baire measures are T-additive (normal in the terminology of [5],[8]). By ([5], Theorems $3.3,3.4)$ every Frechet space is universally measurable and so every $\tau$-smooth measure is tight. This proves the result.

REMARK. In case $E$ is a Banach space, this result is implicit in ([2], $p$. 88(4), Theorem 5.4); if in addition $\mathrm{f}$ is bounded this is proved in ([1], p. 88). 
REFERENCES

1. Diestel, J. and J. J. Uhl Jr. Vector Measures, Amer. Math. Soc. Math. Surveys, Number 15(1977).

2. Edgar, E. Measurability in Banach Spaces, Indiana Univ. Math. J. 26(1977), $663-677$.

3. Khurana, S. S. Strong Measurability in Frechet Spaces, Indian J. Pure Appl. Math. (to appear).

4. Schaefer, H. H. Topological Vector Spaces, Macmillan: New York, 1971.

5. Schwartz, L. Certaines Propriétés des Measures sur les Espaces de Banach, Sem. Maurey-Schwartz, 1975, Ecole Polytechnique, No. 23.

6. Sion, S. On Analytic Sets in Topological Spaces, Trans. Amer. Math. Soc. $96(1960), 341-354$.

7. Talagrand. Sur Une Conjecture de H. H. Corson, Bull. Sci. Math. (2), 99 (1975), 211-212.

8. Varadarajan, V. S. Measures on Topological Spaces, Amer. Math. Soc. Transl. 48(1965), 161-228. 


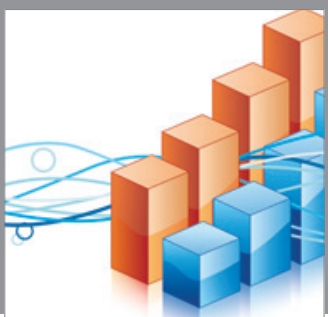

Advances in

Operations Research

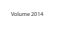

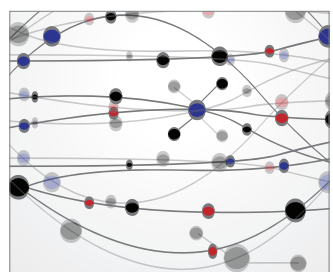

\section{The Scientific} World Journal
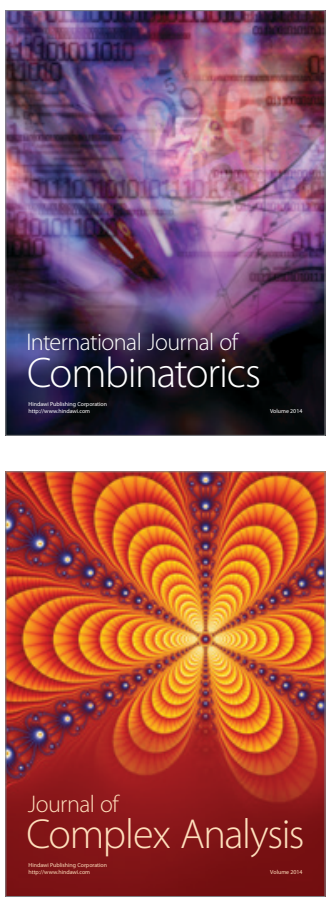

International Journal of

Mathematics and

Mathematical

Sciences
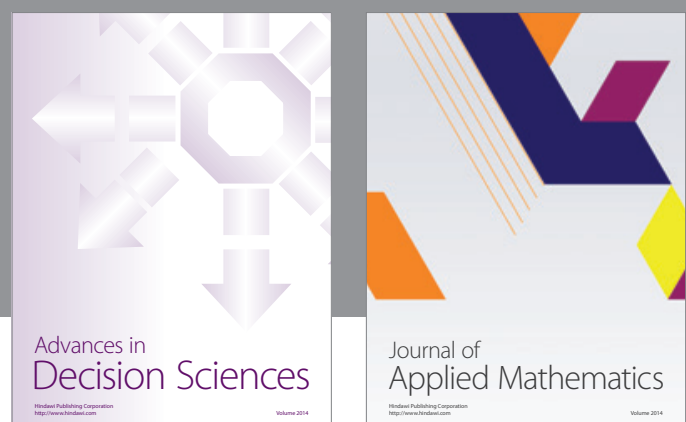

Journal of

Applied Mathematics
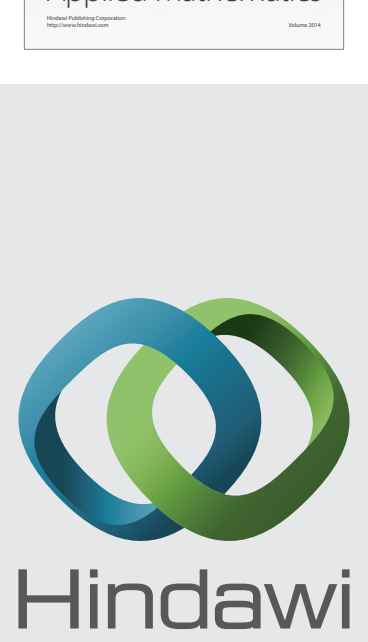

Submit your manuscripts at http://www.hindawi.com
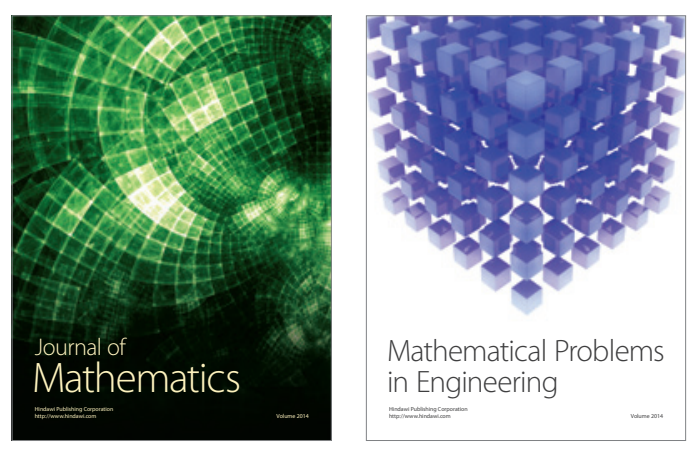

Mathematical Problems in Engineering
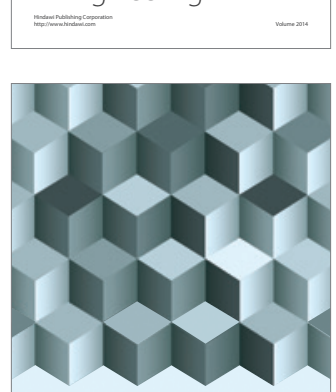

Journal of

Function Spaces
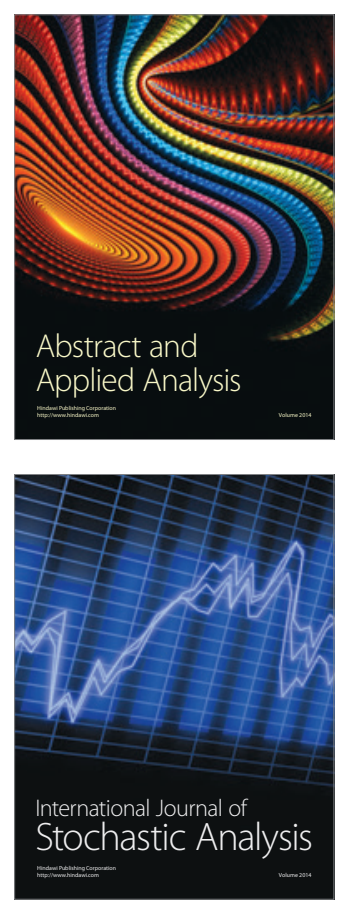

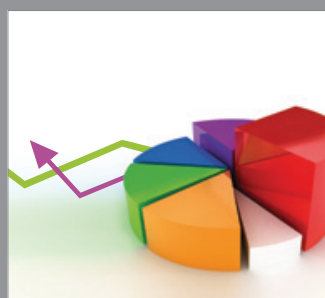

ournal of

Probability and Statistics

Promensencen
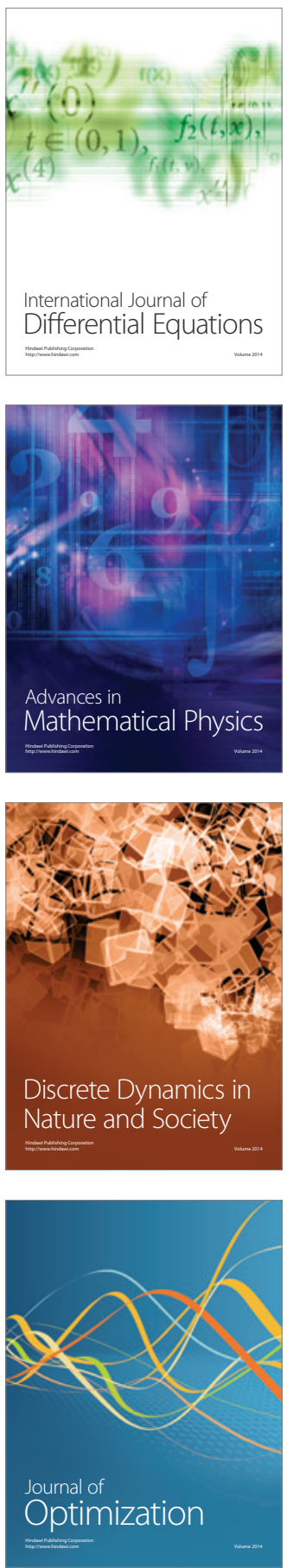